\title{
Examining the evidence base for forensic case formulation: An integrative review of recent research
}

Victoria Wheable* and Jason Davies

Department of Psychology, Swansea University, UK

Correspondence concerning this article should be sent to Victoria Wheable*, Department of Psychology, College of Human and Health Sciences, Vivian Tower, Swansea University, Swansea, SA2 8PP, 967757@swansea.ac.uk

\section{Author Accepted Manuscript}

To cite this article: Wheable, V. \& Davies, J. (2020). Examining the Evidence base for forensic case formulation: An integrative review of recent research. International Journal of Forensic Mental Health. DOI: 10.1080/14999013.2019.1707331 


\section{Examining the evidence base for forensic case formulation: An integrative review of recent research}

In the past decade, forensic case formulation (FCF) has become a key activity in many forensic services. However, the evidence base for FCF remains limited. This integrative review aimed to identify and evaluate all FCF research conducted since the lack of understanding within this field was highlighted by several academics in 2011. A rigorous literature search led to the identification of 14 studies fitting the inclusion criteria. Studies were critically evaluated and synthesised to create a summary of the recent research, to identify remaining gaps in our understanding, and to create an agenda for future research.

Keywords: forensic case formulation, forensic practice, integrative review 


\section{Introduction}

Case formulation has been used in clinical mental health settings for many years as a method of hypothesizing “the causes, precipitants and maintaining influences of a person’s psychological, interpersonal, and behavioural issues” (Eells, 2007, p. 4). Within the past decade, case formulation has now also become an explicit part of work in many forensic services, where it is used to gain an understanding of each offender's criminal behavior, clinical problems and criminogenic needs (Joseph \& Benefield, 2012). This understanding is typically used to devise a tailored management and treatment plan for each offender, with the aim of reducing their risk of re-offending and improving their psychological wellbeing (Minoudis et al., 2013).

In spite of this, a number of academics within the field have highlighted that there is “almost no empirical literature on forensic case formulation” (Sturmey \& McMurran, 2011a, p. 288), and have emphasized the need for research examining the validity, reliability, utility, value and impact of case formulation within forensic services (Hart, Sturmey, Logan, \& McMurran, 2011; Sturmey \& McMurran, 2011b). This research is imperative to conduct, as it has been theorized that the consequences of an inaccurate forensic case formulation (FCF) could include "additional significant adverse outcomes such as repeat serious offending, significant injuries and trauma to others, and large costs of incarceration and long-term treatment” (Sturmey \& McMurran, 2011a, p. 288).

Given the call to action by these academics in 2011, the aim of the present review is to evaluate and synthesize all research ${ }^{1}$ conducted since that time which has specifically aimed to better understand the validity, reliability, quality, utility, value, impact or outcomes of FCF. It is believed that this review will create a better understanding of the current

\footnotetext{
${ }^{1}$ All research conducted in English
} 
evidence base for FCF, will highlight the extent to which imperative questions surrounding the value and impact of FCF have now been answered, and will enable an understanding of how future research should be usefully directed to fill any remaining gaps in our understanding. The conclusions of this review are expected to have implications for the FCF field as a whole.

\section{Method}

\section{Inclusion Criteria and Search Strategy}

An exhaustive search of the FCF literature was carried out between June-August 2018 to identify papers meeting the following inclusion criteria: available in English; involving primary collection of data; concerned specifically with understanding the validity, quality, reliability, value, utility, effectiveness, impact or outcomes of FCF; published since 2011; forensically focused. This search involved the use of electronic databases (PsycINFO, PsycARTICLES, MEDLINE, CINAHL and WoS), hand-searching references within included papers, and contacting known experts within the FCF field. Search terms were entered as follows: 'case formulatio* OR case conceptuali* OR case consultatio* AND forensic OR offend* OR personality disorder OR crim* OR incarcera* OR priso* OR probation OR parole OR correctio*'.

\section{Search Results and Quality Appraisal}

A total of 1,387 records were retrieved. After duplicates were removed, each record was screened for relevance based on its title and abstract. If a record appeared relevant or if its relevance could not be determined, the full text of the article was accessed $(\mathrm{n}=98)$. These articles were then assessed to determine whether they met the stated inclusion criteria. This process resulted in the identification of 14 suitable studies. A second researcher then assessed the relevance of a number of included and excluded 
articles to provide a measure of reliability. No discrepancies emerged. A full summary of the selection and screening process can be viewed in Figure 1.

\section{Figure 1}

About here

\section{Table 1}

About here

\section{Results}

\section{Review Structure}

After further examining the 14 identified studies, it became clear that many of them shared similar research questions and designs. Similar studies were therefore grouped together, revealing 5 distinct categories of research. These categories were then named accordingly; 'The impact of formulation-focused consultation meetings on staff', Opinions on issues within forensic case formulation', 'Forensic case formulation training', ‘Assessing formulation quality in practice', and 'Collaborative forensic case formulation'. Each of these categories will be described, critiqued and evaluated separately to optimize a rich understanding of the research that has been conducted in the FCF field since 2011. These categories will then be combined at the end of the review to facilitate the summation of key findings, to identify overarching gaps within the literature, and to construct an agenda for future research.

\section{The Impact of Formulation-Focused Consultation Meetings on Staff (4 studies)}

\section{Overview.}


Formulation-focused consultation meetings provide a forum for forensic staff to develop a better psychological understanding of offenders and their behavior through a process of discussion and collaboration (Knauer, Walker, \& Roberts, 2017). These consultation meetings have therefore been described as the process of formulating, whereas FCF itself is often seen as the product of this process (NOMS \& NHS, 2015b). Four of the identified studies aimed to assess the impact of formulation-focused consultation meetings on forensic staff (Knauer, Walker, \& Roberts, 2017; McMullan, Ramsden, \& Lowton, 2014; Ramsden, Lowton, \& Joyes, 2014; Whitton, Small, Lyon, Barker, \& Akiboh, 2016).

Formulation-focused consultation meetings within these studies were typically led by a psychologist or caseworker and were attended by an individual member of forensic staff or a team of forensic staff. Bespoke self-report questionnaires were used by Knauer et al., Ramsden et al. and Whitton et al. to measure the impact of these consultation meetings on staff understanding of offenders, staff competence in working with offenders, and staff attitudes toward offenders. Ramsden et al. additionally used the Personality Disorder Knowledge, Attitudes and Skills Questionnaire (PDKASQ; Bolton, Feigenbaum, Jones, Sims, \& Woodward, 2010) to measure change within these areas.

Staff within these studies were either assessed before and after a 3-month period of ongoing consultation meetings (Ramsden et al., 2014), or directly before and after attending a single consultation meeting (Knauer et al., 2017; Whitton et al., 2016). Knauer et al. additionally assessed staff a third time after they had received a written FCF letter summarizing the meeting they had attended. The fourth study (McMullan et al., 2014) instead used qualitative methods to understand staff views and opinions of an ongoing process of consultation meetings. Some of the staff within this study were still part of this process, whereas others had withdrawn.

The results of all four studies indicated that formulation-focused consultation meetings 
increased staff understanding of offenders. Improvements were also found in staff knowledge of offenders, staff confidence in managing offenders, staff motivation to work with offenders and staff satisfaction with pathway plans (Knauer et al., 2017), capability of working with personality disordered offenders and positive emotions toward these offenders (Ramsden et al., 2014), psychological understanding and team consistency (Whitton et al., 2016), and awareness (McMullan et al., 2014).

Less positively, a quarter of participants $(n=3)$ within the study by McMullan et al. described the ongoing process of consultation meetings as “frustrating” (p. 189). Knauer et al. also found that receiving a written FCF letter summarizing the content of the meeting did not further improve staff outcomes significantly. In contrast however, several staff within the study conducted by McMullan et al. indicated that they would find it helpful for the content of the meetings to be "written up into a report” (p. 191).

\section{Strengths.}

The consultation meetings that took place within these studies were part of routine practice and the staff that attended these were recruited through naturalistic methods. These factors suggest that these four studies have good ecological validity. Additionally, staff from a wide range of forensic services were involved, including those from approved premises, probation, prison and secure wards settings. McMullan et al. also included staff who had previously withdrawn from the consultation meeting process. Together, these factors suggest the participants recruited are highly representative of the wider workforce.

\section{Limitations.}

The bespoke questionnaires and PDKASQ used to measure staff outcomes are self-report measures, which could be subject to biased or socially desirable responding. In addition, research on the PDKASQ has suggested that it has low construct validity (Shaw, Minoudis, Craissati, \& Bannerman, 2012). This indicates that although staff reported improvements in 
areas such as understanding, capability and knowledge with the use of these measures, it is not known whether these improvements were genuine or would have led to positive improvements in practice.

Attrition rates were also high within a number of the studies. This was most evident within the Ramsden et al. study, in which $74 \%$ of participants withdrew before completing the final assessment of their outcomes. This resulted in only 12 participants completing the PDKASQ and 6 completing the bespoke questionnaire after the 3-month consultation meeting process had finished. Due to this, independent t-tests appear to have been inappropriately performed in place of paired t-tests for PDKASQ scores, and no statistical analysis was performed on the bespoke questionnaire responses. Additionally, only $25 \%$ of the initial participants took part in the third phase of the Knauer et al. study (15 vs 60), suggesting that the absence of further staff improvement after receiving a written FCF letter could be attributable to a depleted sample size.

A final limitation to note is that within the study by Whitton et al., a number of participants indicated that they had never met the offender discussed within the consultation meeting. It would therefore be expected that scores on items such as "I have a good psychological understanding of the patient’s problems” (p. 156) would increase significantly after this meeting. This may have led to the benefits attributed to consultation meetings within this study being unwittingly inflated.

\section{Conclusions.}

Although several limitations have been discussed above, the repeatedly positive results found by these studies suggest that consultation meetings do have a positive impact on the outcomes of forensic staff. There are however a few areas that require further clarification. One of the studies reviewed here found that producing a written FCF which summarized the content of the consultation meeting did not produce any additional benefit for staff. However, it is 
recognized that written FCF may have effects and functions above and beyond its impact on staff (e.g., providing a document for continuity of care; various impacts on the offender). In addition, within some forensic services such as the Offender Personality Disorder Pathway (OPDP), written FCFs are already developed for each offender which takes up time and other valuable resources. Thus, further research on the value and impact of written FCF is required.

Secondly, these four studies encouragingly suggest that consultation meetings are able to contribute to meeting one of the core aims of the OPDP: workforce development. However, future research needs to adopt validated measures and must examine the potential impact of consultation and written FCF on outcomes other than self-reported staff improvements. This could include understanding whether these self-reported improvements lead to positive changes in staff practice, and whether this then leads to more positive outcomes for offenders.

\section{Opinions on issues within forensic case formulation (3 studies)}

\section{Overview.}

Whilst case formulation has traditionally been the domain of psychologists and psychiatrists (Division of Clinical Psychology, 2011), offender managers (OMs) within the OPDP are now expected to play a prominent role in the construction of FCFs (NOMS \& NHS, 2015a). To understand how OMs, personality disordered (PD) offenders, and carers of PD offenders felt about OMs carrying out FCF within this service, Brown and Völlm (2013; 2016,) conducted a series of focus groups. Völlm (2014) instead used a Delphi method to gain expert consensus on issues such as who should carry out FCF for PD offenders, what it should include and how its quality should be measured. Experts were defined as those who had a background in psychology/psychiatry and who had recent experience of working with PD offenders or had recently published work in the PD and/or offending field.

Participants within all three studies raised concerns about whether OMs could successfully carry out FCF within the OPDP (Brown \& Völlm, 2013; 2016; Völlm, 2014). 
OMs and experts generally believed that OMs would be capable of this task if in-depth training was provided to them (Brown \& Völlm, 2013, Völlm, 2014), but offenders and carers believed that any OM training would be necessarily short and therefore ineffective (Brown \& Völlm, 2016). Offenders additionally raised concerns about the dual role of OMs, overseeing “punishment” and also now “care” (p. 221).

Experts could not reach consensus on several issues. This included how to best to assess the quality of a FCF, with some comments highlighting the lack of a valid and reliable quality measure (Völlm, 2014). In addition, only 40\% of experts agreed that offenders who had received a FCF would have more positive outcomes. The remainder of experts felt that they were not able to accurately judge this, with some citing the lack of evidence confirming the effectiveness of FCF.

\section{Strengths.}

This opinion research succeeded in recruiting a wide variety of participants beyond forensic staff. This enabled a greater understanding of how FCF is viewed by all those who may be affected by its use and has revealed important differences in these views. Furthermore, some of the results of these opinion studies have already resulted in action. For example, based on the view that further OM FCF training was needed (Brown \& Völlm, 2013; Völlm, 2014), a training package was later developed and delivered to OMs in a study by Brown, Beeley, Patel and Völlm (2018). This study is discussed later in the "Forensic case formulation training” section of this review.

\section{Limitations.}

Despite the diversity of participants recruited into these studies and the inclusion of 'hard to reach' populations (e.g., high-risk offenders), the sample sizes were relatively small. For example, Brown and Völlm (2016) conducted only two offender focus groups, with a third canceled due to access difficulties. Some sources however suggest that at least three focus 
groups are needed to extract prevalent themes (Guest, Namey \& McKenna, 2017). In addition, a high attrition rate resulted in only 54\% of the experts within the Völlm et al. study completing the second round of the Delphi survey. Together, these issues are likely to have reduced the generalizability of the findings.

It is also important to note that all three of these opinion studies were carried out by the same two authors (Brown \& Völlm, 2013; 2016; Völlm, 2014). It is therefore possible that the participant pool accessible to these researchers was limited.

\section{Conclusions.}

The findings of this small body of research were mixed; some participants felt optimistic about OMs carrying out FCF within the OPDP, whereas others were skeptical. However, these studies were conducted when FCF was first implemented into the OPDP, and so many of the opinions expressed may have been magnified by uncertainty relating to this change. OMs, offenders, carers and experts may now feel very differently about these issues and so it would be of value for further research to be undertaken in this area. Concerning the findings of Völlm (2014), research is sorely needed to investigate how FCF might impact offender outcomes. Once this is understood, it may be easier to understand what an effective and therefore 'high-quality' formulation consists of so that a valid quality measure can be developed.

\section{Forensic case formulation training (4 studies)}

\section{Overview.}

Four of the identified studies aimed to understand whether the FCF skills of OMs working within the OPDP could be improved through training. Two of these studies (Brown, Beeley, Patel, \& Völlm, 2018; Mapplebeck, Ramsden, Lowton, Short, \& Burn, 2017) did this by assessing OM formulation skills both before and immediately after training. Minoudis et al. (2013) instead assessed OM formulation skills before and after they had attended both a 
period of training and a number of formulation-focused consultation meetings in practice. The formulations resulting from these consultation meetings were also assessed by the researchers. The fourth study (Radcliffe, McMullan, \& Ramsden, 2018) compared the formulation skills of OMs who had received OPDP training and ongoing supervision in practice for 6 months to 3 years with OMs who had not received this training or supervision.

Although the content of the training itself was similar across studies (mainly focusing on FCF and understanding PD), the duration of the training varied. OMs within the Minoudis et al. study were provided with only 8 hours of initial training, whereas OMs in the other three studies were provided with five or six days of training.

OMs within the Minoudis et al. study used one of two fictitious case vignettes to construct a formulation both before and after completing their 8 hours of training and attending a number of consultation meetings in practice (OMs used one vignette at baseline and the other vignette during the final assessment). The formulations constructed during the consultation meetings they had attended were however carried out on real cases. OMs within the studies conducted by Brown et al. and Radcliffe et al. used the same two fictitious case vignettes developed by Minoudis et al. to construct their formulations.

Brown et al. and Minoudis et al. assessed these formulations using the Case Formulation Quality Checklist (CFQC; McMurran, Logan, \& Hart, 2012), whereas Radcliffe et al. assessed formulations using the Formulation Quality Checklist (NOMS \& NHS, 2015b). Brown et al. additionally asked OMs to complete the PDKASQ before and after training. OMs within the qualitative study by Mapplebeck et al. were instead shown one fictitious case vignette both before and after training and were simply asked what additional information they would request about this case and what they would focus on if this offender was on their caseload.

The findings of these training studies were mixed. Brown et al., Radcliffe et al. and 
Mapplebeck et al. all concluded that either training alone or training plus supervision in practice had a positive impact on the FCF skills of OMs. Minoudis et al. however found that OMs showed no significant improvement in their formulation skills even after 8 hours of training and a 6-month period of attending formulation-focused consultation meetings in practice. This might suggest that the length of the initial training period is the most important factor, potentially indicating that OM formulation skills cannot be significantly developed in practice without firstly providing them with a firm foundation of knowledge. It is however also noted that OMs within the Minoudis et al. study had significantly poorer baseline formulation skills on average (14.2/40) than those within the Brown et al. study (24.8/40) as rated by the CFQC. This may therefore have magnified the effect of this shorter training period.

\section{Strengths.}

A strength of these four studies concerns the likely quality of the training delivered to OMs. The studies by Radcliffe et al. and Mapplebeck et al. assessed the impact of routine OPDP induction training, Brown et al. developed their training based on previous research (Brown \& Völlm, 2013; Völlm, 2014) and on an educational program co-commissioned by the Department of Health and the Ministry of Justice (Baldwin, 2011), and training within the study by Minoudis et al. was developed by two experienced chartered psychologists aided by a range of academic sources. This suggests that the mixed findings of these studies are unlikely to be due to quality differences in the training provided to OMs.

Additionally, the fictitious case vignettes used within the three quantitative studies were identical, based on those developed by Minoudis et al. As noted by Radcliffe et al., this helps to mitigate any confounding factors that could result from using different vignettes. Differences in the findings of these studies are therefore likely to be due to intentional manipulations in the length of training or ongoing development period rather than 
unintentional differences in vignette quality or complexity.

Finally, Minoudis et al. also assessed some of the psychometric properties of the CFQC within their study and concluded it had moderate to good inter-rater reliability, excellent test-re-test reliability and excellent internal validity. This suggests it was an appropriate outcome measure to use.

\section{Limitations.}

Minoudis et al., who developed the vignettes used within the three quantitative studies, describe them as “necessarily brief” (p. 260) and acknowledge that the quantity and quality of information contained within them may not be equal to that available when formulating a case in practice. Similarly, the vignette developed and used by Mapplebeck et al. was also described as "brief” (p. 38). This may have negatively impacted the ecological validity of some of the training findings. In addition, Mapplebeck et al. used the same vignette both before and after training, suggesting that the small qualitative improvements observed posttraining could have been the result of practice effects caused by OMs already being familiar with this material and having had time to think about its content before being assessed a second time.

Secondly, although Minoudis et al. assessed some of the psychometric properties of the CFQC within their study, the predictive validity of both the CFQC and the Formulation Quality Checklist remains unknown. Therefore, until it is understood whether higher scores on these tools relate to more positive outcomes, it cannot be confirmed whether these tools are providing an accurate measure of formulation quality. This suggests that we must endeavor to validate these tools before we can truly understand whether the FCF skills of OMs can be improved in any meaningful way.

Finally, although three of the studies concluded that OM FCF skills improved after either training alone or training plus supervision in practice, the extent of these improvements 
was variable. For example, Brown et al. found that OM formulations significantly improved on 7 items of the CFQC after training, but no significant change was found in 'simplicity', 'external consistence' or 'action oriented'. This suggests that the post-training formulations completed by OMs were still not consistent with psychological theory and continued to lack information about treatment selection and planning. In addition, Mapplebeck et al. concluded that although OMs focused more heavily on the psychological aspects of a case after training, this change was not observed in the domain of offending behavior and risk. These findings are important to consider, as one of the main purposes of FCF is to create a psychological understanding of an individual's offending behavior which can be used to construct an appropriate plan of management and treatment.

\section{Conclusions.}

The majority of these findings were positive (Brown et al., 2018; Mapplebeck et al., 2017; Radcliffe et al., 2018), suggesting that training over several days, possibly with a subsequent period of application, can improve the FCF skills of OMs. This may resolve some of the concerns raised within the opinion research described earlier (Brown \& Völlm, 2013; 2016; Völlm, 2014). However, these findings should be regarded cautiously due to the methodological drawbacks discussed.

Further research is needed to fully validate the tools used to measure FCF quality before firm conclusions can be made about the impact of OM training. Researchers should also consider using authentic case information during training instead of brief vignettes to understand if OMs are able to develop the skills needed to formulate complex cases. Finally, research should be conducted to better understand how differences in baseline FCF skills, length of formal training, and length of post training experience can influence the quality of OM formulations.

\section{Assessing formulation quality in practice (2 studies)}




\section{Overview.}

Two of the identified studies focused on assessing and understanding the quality of formulations developed by psychologists in practice (McMurran \& Bruford, 2016; Hopton, Cree, Thompson, Jones, \& Jones, 2018).

McMurran and Bruford gathered feedback on the utility of the CFQC from 10 clinicians who had used it in practice. These clinicians reported that the CFQC was a useful, comprehensive and appropriate quality assessment tool, but questioned its inter-rater reliability, complex language and restrictive Likert scales used for scoring items. From this feedback, the authors developed the Case Formulation Checklist Revised (CFQC-R), featuring simplified language and expanded Likert scales.

The CFQC-R was then used by Hopton et al. to measure the quality of a number of risk formulations (RF) constructed by psychologists within forensic psychiatric hospitals. Each of these RFs had been constructed as part of either version 2 or version 3 of the Historical Clinical Risk-20 (HCR-20) tool. The latter version of this tool has a larger focus on formulation, and so it was hypothesized that RFs constructed with this version may be significantly better in quality than those constructed with the earlier version. To clarify, RFs are similar to FCFs but are narrower in focus, concentrating specifically on better understanding, explaining and managing an offender's risk of harm to themselves and others. As this definition is very similar to that of FCF, this study met the inclusion criteria for the present review.

Although RFs carried out using version 3 of the HCR-20 were found to be of better quality than those carried out using version 2, it was found that overall, the RFs were generally of poor to intermediate quality as rated by the CFQC-R. This was mainly due to being overly descriptive and having little focus on constructing hypotheses, making predictions about future behavior, or developing treatment plans. Hopton et al. additionally 
assessed the inter-rater reliability of the CFQC-R and found it to be good.

\section{Strengths.}

A major strength of both of these studies is that they appear to have a high level of ecological validity. McMurran and Bruford recruited clinicians who had already used the CFQC in routine practice, indicating that these clinicians were able to give informed opinions on the clinical utility of the tool and that these opinions were uninfluenced by the researchers. Hopton et al. randomly selected 121 completed RFs from 17 forensic psychiatric hospitals, ensuring firstly that the construction of these RFs was not influenced by the presence of the researchers, and secondly that the findings obtained from this research are likely to be highly representative of RFs completed across these services. In addition, both studies focused on the quality assessment of formulations completed by psychologists. This type of research may be helpful in establishing a realistic standard of FCF quality against which the skills of other populations can be compared (e.g., OMs).

\section{Limitations.}

Although clinicians within the McMurran and Bruford study reported the CFQC to be a useful and appropriate quality tool to use and Hopton et al. found the CFQC-R to have good interrater reliability, the predictive validity of both of these tools is yet to be confirmed. A consequence of this is that although Hopton et al. concluded that RFs completed by psychologists within a number of forensic psychiatric hospitals were of poor to intermediate quality, this does not necessarily mean that they were, or that they would have been any less likely to have a positive impact on outcomes than RFs scoring highly on this tool.

Furthermore, the CFQC-R was developed for use specifically with FCFs, and so it is not known how well it may also apply to RFs. For example, although the RFs scored poorly on the criteria of 'action oriented', Hopton et al. explain that this may be because treatment plans are often recorded within another section of the HCR-20 which was not analyzed within 
their study. This suggests that the use of the CFQC-R within this study may have created the impression that the RFs were of poorer quality than they truly were.

An additional limitation relates to the methods of data collection used by McMurran and Bruford. Clinicians within this study were interviewed either in person, via telephone or via email. However, telephone interviews lasted 16.5 minutes on average, whereas interviews in person lasted 32 minutes on average. This suggests that participants interviewed in person had the opportunity to provide much more data than others, potentially skewing the results. It is also reported that notes were made during the interviews and written up later "as close to verbatim as possible” (p.33). The interviewer may have therefore inadvertently noted more points which they found personally interesting or relevant, which could have impacted the conclusions drawn.

\section{Conclusions.}

Although staff within the McMurran and Bruford study reported the CFQC to be a useful tool, the utility of this finding is limited due to outstanding questions surrounding the predictive validity of this tool. While the CFQC-R also suffers from this limitation and in addition may not be well suited to assessing RFs, it would be unwise to overlook the findings of Hopton et al. (2018). This is because the RFs assessed within this study also scored poorly on a number of items which they would have been expected to score more highly on. These items include 'external coherence' (the formulation is consistent with an empirically supported theory) and 'completeness' (the formulation ties together as much of the relevant information as possible). This suggests that the formulation skills of psychologists should also be further investigated to understand how these are developed and updated over time.

\section{Collaborative forensic case formulation (1 study)}

\section{Overview.}

Although not necessarily typical of formulation within forensic settings, FCFs within the 
OPDP are often carried out non-collaboratively, meaning that they are constructed about offenders rather than with offenders (Shaw, Higgins, \& Quartey, 2017). However, the clinical literature suggests that collaborative formulation may be more beneficial than non-collaborative formulation (Division of Clinical Psychology, 2011; Kuyken, 2006; Kuyken, Padesky, \& Dudley, 2008; Persons, 1989).

On the basis of this, one study within the present review aimed to investigate the impact of collaborative FCF on OMs and offenders within the OPDP (Shaw, Higgins and Quartey, 2017). Firstly, OMs were randomly allocated into either a collaborative or noncollaborative FCF condition. Those within with the collaborative condition were then provided with advanced collaborative FCF training. After this, all OM's were randomly allocated an eligible offender and were asked to construct a FCF for this offender within routine practice (collaboratively or non-collaboratively based on condition). After 20 weeks, OMs and offenders were asked to complete the Dual Role Relationships Inventory - Revised (DRI-R; Skeem, Louden, Polaschek, \& Camp, 2007), which is a self-report measure of the quality of OM-offender relationships. OMs also completed the Perceived Benefits Rating Scale (PBRS) which was developed specifically for the study by Shaw et al. This scale aimed to assess OM confidence in managing their allocated offender and to assess OM perceptions of their allocated offenders' outcomes, including their level of compliance with their sentence plan and their level of motivation to cease offending.

Positively, offenders within the collaborative condition reported significantly higher DRI-R 'Trust' scores than offenders within the non-collaborative condition, suggesting they felt higher degrees of trust in their OM. OMs within the collaborative condition reported significantly higher DRI-R ‘Caring and Fairness’ scores (which Shaw et al. suggest broadly translates into feeling a stronger working alliance with their allocated offender) and significantly higher confidence in managing their offender than OMs within the non- 
collaborative condition. OM perceptions of offender outcomes however did not significantly differ between conditions.

\section{Strengths.}

A strength of this study is that that some aspects of its methodology were designed very rigorously. Clear inclusion criteria were specified for those taking part, and a range of potentially confounding variables were identified and controlled for. The randomized design used and targeted analyses performed rule out the likelihood of the results found being due to differences in OM or offender characteristics between conditions.

\section{Limitations.}

Although the 77 OMs recruited into the study were allocated roughly evenly into each condition, only 13 offenders took part in the collaborative condition (due to consent difficulties), whereas 26 offenders took part in the non-collaborative condition. This suggests that all collaborative formulations were constructed by more than one OM, potentially influencing offender and OM outcomes within this condition.

Additionally, OM drop out was relatively high and not evenly distributed across groups (collaborative condition: 29.7\%; non-collaborative condition: 20\%). Two OMs within the collaborative condition dropped out due to withdrawal of consent, whereas all OMs who dropped out from the non-collaborative condition did so due to reasons unrelated to the study (e.g., maternity leave and sickness). If those who withdrew their consent did so because of problematic OM-offender relationships, this could have significantly skewed the findings.

The advanced collaborative formulation training provided to OMs within the collaborative condition may have also inadvertently affected outcomes. This is because Shaw et al. state that prior to the study, all OM's had previously completed only half a day of basic formulation training. This suggests that the extra training provided to OMs within the collaborative condition may have enhanced their existing formulation skills, rather than 
simply equipping them with separate collaborative formulation skills. This may have given OMs in the collaborative condition an unfair advantage and would suggest that it could be this training, rather than the collaborative formulation, which produced the positive effect on OMoffender relationships within this condition.

Finally, the PBRS was developed by Shaw et al. for the purposes of the study and has therefore not been validated. It is also a measure of OM perceptions of offender outcomes, which may not accurately reflect the true outcomes of these offenders. The results obtained with the use of this scale should therefore be viewed with caution.

\section{Conclusions.}

Although this study is useful in its attempt to compare the benefits of collaborative versus non-collaborative FCF within the OPDP, it is difficult to come to any firm conclusions due to the limitations noted. Future research within this area should aim to address these limitations and should also attempt to measure actual rather than perceived offender outcomes. The finding that collaborative formulation may have the ability to significantly improve OMoffender relationships is however very encouraging and suggests that carrying out formulations collaboratively within the OPDP should be considered.

\section{Discussion}

\section{Summary of research findings}

The FCF research conducted since 2011 reveals a number of promising findings, suggesting that formulation-focused consultation meetings are beneficial to forensic staff (Knauer, Walker, \& Roberts, 2017; McMullan, Ramsden, \& Lowton, 2014; Ramsden, Lowton, \& Joyes, 2014; Whitton, Small, Lyon, Barker, \& Akiboh, 2016), that training alone or training plus ongoing supervision in practice can improve the FCF skills of OMs (Brown, Beeley, Patel, \& Völlm, 2018; Mapplebeck, Ramsden, Lowton, Short, \& Burn, 2017; Radcliffe, McMullan, \& Ramsden, 2018), that clinicians find the CFQC to be a useful and appropriate 
measure of formulation quality (McMurran \& Bruford, 2016), and that carrying out collaborative FCFs within the OPDP can positively impact OM-offender relationships (Shaw, Higgins, \& Quartey, 2017). Less positive findings of this research however include that there is some skepticism about OMs carrying out FCF within the OPDP (Brown \& Völlm, 2013; 2016; Völlm, 2014), that experts cannot reach consensus on some fundamental issues within FCF (Völlm, 2014), that OM training and development may not always be associated with improvements in FCF skills (Minoudis et al., 2013), and that RFs constructed by psychologists may be of poor to intermediate quality (Hopton et al., 2018). This summary highlights that much of the FCF research conducted since 2011 has concentrated either on understanding self-reported staff outcomes of consultation/FCF or measuring/improving FCF quality.

\section{Gaps in the research and homogeneity of the research}

Although some progress has been made in the FCF field since the call to action by a number of academics in 2011 (i.e., Hart, Sturmey, Logan, \& McMurran, 2011; Sturmey \& McMurran, 2011a) as highlighted above, our understanding of the value, impact and outcomes of FCF remains limited. This is likely due to the narrow scope of the research, with 12 of the 14 reviewed studies aiming to investigate the skills, understanding, knowledge or opinions of forensic staff members in relation to consultation meetings or FCF (Brown, Beeley, Patel, \& Völlm, 2018; Brown \& Völlm, 2013; Hopton, Cree, Thompson, Jones, \& Jones, 2018; Knauer, Walker, \& Roberts, 2017; Mapplebeck, Ramsden, Lowton, Short, \& Burn, 2017; McMullan, Ramsden, \& Lowton, 2014; McMurran \& Bruford, 2016; Minoudis et al., 2013; Radcliffe, McMullan, \& Ramsden, 2018; Ramsden, Lowton, \& Joyes, 2014; Völlm, 2014; Whitton, Small, Lyon, Barker, \& Akiboh, 2016), rather than exploring the impact of these processes on other populations of interest such as offenders.

In addition, the present review has revealed that although a number of studies 
conducted within the FCF field since 2011 have focused on better understanding and assessing FCF quality (Brown, Beeley, Patel, \& Völlm, 2018, Hopton, Cree, Thompson, Jones, \& Jones, 2018; McMurran \& Bruford, 2016; Minoudis et al., 2013; Radcliffe, McMullan, \& Ramsden, 2018), each of these studies have incorporated the use of unvalidated quality measures. The implication of this is that the findings of these studies may be unreliable, further limiting our understanding of quality within the FCF field.

These issues of narrow scope and similar methodological limitations may be explained by a number of factors. The first is that a very high concentration of this research focused on investigating FCF within the OPDP specifically, rather than a wider range of forensic services. This is likely to be why many of the studies focused on the skills, understanding and knowledge of forensic staff in particular, as OMs within the OPDP are expected to play a large role in the construction of FCFs. In addition, 11 out of the 14 studies involved the input of one of just four authors; McMurran, Ramsden, Shaw or Völlm (Brown, Beeley, Patel, \& Völlm, 2018; Brown \& Völlm, 2013; 2016; Mapplebeck, Ramsden, Lowton, Short, \& Burn, 2017; McMullan, Ramsden, \& Lowton, 2014; McMurran \& Bruford, 2016; Minoudis et al., 2013; Radcliffe, McMullan, \& Ramsden, 2018; Ramsden, Lowton, \& Joyes, 2014; Shaw, Higgins, \& Quartey, 2017; Völlm, 2014), which may explain the similarity of the topics investigated. Increased diversity is therefore needed in the research questions being asked and the range of methodologies being used in order to enable the further development of knowledge and understanding within the FCF field.

Together, these points indicate that although the need for research examining the validity, reliability, quality, utility, value, impact and outcomes of case formulation within forensic services was highlighted almost a decade ago, our understanding of these fundamental issues remains limited.

\section{Future directions.}


In view of the points made above, there are several areas where future research attention could be usefully directed. The first would be to investigate the outcomes of consultation meetings and FCFs beyond those perceived by forensic staff. This could include investigating potential impacts on the offender, such as changes in risk or psychological well-being. If positive impacts of this nature were observed, future research could then provide an understanding of the mechanism by which consultation meetings or FCFs are working to impact these outcomes. For example, consultation meetings or FCFs may initially improve staff understanding and knowledge of offenders, enabling these staff members to manage offenders more effectively. The results of this type of research could have a large positive impact in practice, as providing staff with a full understanding the benefits of consultation and FCF may motivate them to fully utilize these resources.

A second important direction for future research concerns the empirical understanding of formulation quality. As discussed above, studies investigating FCF quality within the present review have repeatedly incorporated the use of unvalidated quality tools to measure the FCF skills of psychologists and OMs, rather than aiming to develop a comprehensive understanding of what FCF quality genuinely comprises of. However, by linking FCF to offender outcomes, it may be possible to develop this comprehensive understanding relatively easily. For instance, by examining the formulations of offenders who have achieved positive outcomes (no re-offending, improved psychological wellbeing), it may be possible to identify reoccurring features, characteristics or components within these formulations. This identification of important formulation features could have the ability to facilitate a greater understanding of 'what works' within FCF, leading to the development of a fully evidenced quality tool to be used by staff in practice.

An alternative method of developing a more comprehensive understanding of formulation quality could be to score formulations against criteria included within existing 
FCF quality tools used throughout the literature (CFQC, CFQC-R and Formulation Quality Tool). Scores on these tools could then be linked with the outcomes of offenders to identify any patterns of interest (i.e., high scores on item 1 of the CFQC-R are associated with improved psychological wellbeing). This method would provide both an understanding of the predictive validity of these quality tools and also a method of confirming or disputing the findings of studies exploring formulation quality within the present review. Although currently unvalidated, these tools consist of criteria which represent our best understanding of what is important to include within a FCF. The use of these tools may therefore provide a starting point in identifying important formulation features before this search is then expanded. In sum, formulation quality must be fully understood before it can be accurately interpreted, assessed or compared. Without this understanding, the results of research conducted within other areas of interest (i.e., measuring the formulation skills of psychologists and OMs) is likely to produce further unreliable findings.

\section{Review implications}

The present review has highlighted that since the call to action by a number of academics in 2011 (i.e., Hart, Sturmey, Logan, \& McMurran, 2011; Sturmey \& McMurran, 2011a), a number of studies have been carried out within the FCF field. However, many of these studies have sought to answer a small amount of very similar research questions, resulting in the findings of this research feeling relatively limited overall. This suggests that although it was stated in 2011 that the consequences of an inaccurate FCF could include "additional significant adverse outcomes such as repeat serious offending, significant injuries and trauma to others, and large costs of incarceration and long-term treatment” (Sturmey \& McMurran, 2011a, p. 288), we still do not have a full understanding of the validity, quality or impact of FCF within forensic services. In addition to this, large amounts of time, money and resources are being spent every year carrying out FCFs within these services. 
Therefore, although it is recognized that future research in the directions specified above would require more complex, longer term and potentially more expensive designs, this type of research is imperative to conduct as first highlighted almost a decade ago. If this research indicates that FCF is not having the impact intended, this would suggest that resources may be more effectively spent elsewhere. 


\section{References}

Baldwin, V. (2011). Yorkshire \& the Humber KUF Evaluation Report. 2010-2011. Nottingham: Institute of Mental Health.

Bolton, W., Feigenbaum, J., Jones, A., Sims, A., \& Woodward, C. (2010). Development of the PD-KASQ (personality disorder-knowledge, attitudes and skills questionnaire). London: Oscar Hill Service, Camden and Islington NHS Foundation Trust.

Brown, S., Beeley, C., Patel, G., \& Völlm, B. (2018). Training probation officers in case formulation for personality disordered offenders. Criminal Behaviour and Mental Health, 28(1), 50-60. doi:10.1002/cbm.2006 .

Brown, S., \& Völlm, B. (2013). Case formulation in personality disordered offenders: Views from the front line. Criminal Behaviour and Mental Health, 23(4), 263-273. doi:10.1002/cbm.1887

Brown, S., \& Völlm, B. (2016). The implementation of case formulation by probation officers: service user and carer views. The Journal of Forensic Psychiatry \& Psychology, 27(2), 215-231. doi:10.1080/14789949.2015.1122821

Division of Clinical Psychology. (2011). Good Practice Guidelines on the use of psychological formulation. Leicester, UK: British Psychological Society.

Eells, T. D. (2007). Psychotherapy case formulation: History and current status. In T. D. Eeels (Ed.), Handbook of Psychotherapy Case Formulation (2nd ed., pp. 3-32). New York: Guildford Press.

Guest, G., Namey, E., \& McKenna, K. (2016). How Many Focus Groups Are Enough? Building an Evidence Base for Nonprobability Sample Sizes. Field Methods, 29(1), 3-22. doi:10.1177/1525822X16639015

Hart, S., Sturmey, P., Logan, C., \& McMurran, M. (2011). Forensic Case Formulation. International Journal of Forensic Mental Health, 10(2), 118-126. doi:10.1080/14999013.2011.577137

Hong, Q. N., Pluye, P., Fàbregues, S., Bartlett, G., Boardman, F., Cargo, M., . . Vedel, I. (2018). Mixed Methods Appraisal Tool (MMAT), version 2018. Retrieved from http://mixedmethodsappraisaltoolpublic.pbworks.com/w/file/127916259/MMAT 2018 crit eria-manual 2018-08-01 ENG.pdf

Hopton, J., Cree, A., Thompson, S., Jones, R., \& Jones, R. (2018). An evaluation of the quality of hcr20 risk formulations: A comparison between hcr-20 version 2 and hcr-20 version 3 . The International Journal of Forensic Mental Health, 17(2). doi:10.1080/14999013.2018.1460424

Joseph, N., \& Benefield, N. (2012). A joint offender personality disorder pathway strategy: An outline summary. Criminal Behaviour and Mental Health, 22(3), 210-217. doi:10.1002/cbm.1835

Knauer, V., Walker, J., \& Roberts, A. (2017). Offender personality disorder pathway: the impact of case consultation and formulation with probation staff. The Journal of Forensic Psychiatry \& Psychology, 28(6), 825-840. doi:10.1080/14789949.2017.1331370

Kuyken, W. (2006). Evidence-based case formulation: Is the emperor clothed? In N. Tarrier (Ed.), Case formulation in cognitive behaviour therapy: The treatment of challenging and complex cases (pp. 12-35). New York, NY: Routledge.

Kuyken, W., Padesky, C. A., \& Dudley, R. (2008). The Science and Practice of Case Conceptualization. Behavioral and Cognitive Psychotherapy, 36(6), 757-768. doi:10.1017/s1352465808004815

Mapplebeck, C., Ramsden, J., Lowton, M., Short, S., \& Burn, F. (2017). Embedding psychological thinking: an evaluation of a regional training model for probation staff. The Journal of Forensic Practice, 19(1), 37-46. doi:10.1108/JFP-12-2015-0053

McMullan, E., Ramsden, J., \& Lowton, M. (2014). Offender personality disorder pathway: Evaluation of team consultation. Mental Health Review Journal, 19(3), 185-195. doi:10.1108/MHRJ-122013-0041

McMurran, M., \& Bruford, S. (2016). Case formulation quality checklist: a revision based upon clinicians' views. Journal of Forensic Practice, 18(1), 31-38. doi:10.1108/JFP-05-2015-0027

McMurran, M., Logan, C., \& Hart, S. (2012). Case formulation quality checklist, unpublished checklist. 
Minoudis, P., Craissati, J., Shaw, J., McMurran, M., Freestone, M., Chuan, S. J., \& Leonard, A. (2013). An evaluation of case formulation training and consultation with probation officers. Criminal Behaviour and Mental Health, 23(4), 252-262. doi:10.1002/cbm.1890

Moher, D., Liberati, A., Tetzlaff, J., Altman, D. G., \& The Prisma Group. (2009). Preferred Reporting Items for Systematic Reviews and Meta-Analyses: The PRISMA Statement. PLOS Medicine, 6(7), e1000097. doi:10.1371/journal.pmed.1000097

National Offender Management Service, \& NHS England. (2015a). The Offender Personality Disorder Pathway Strategy 2015. Retrieved from https://www.england.nhs.uk/commissioning/wpcontent/uploads/sites/12/2016/02/opd-strategy-nov-15.pdf

National Offender Management Service, \& NHS England. (2015b). Working with offenders with personality disorder: A practitioners guide. Retrieved from https://www.england.nhs.uk/commissioning/wp-content/uploads/sites/12/2015/10/workoffndrs-persnlty-disorder-oct15.pdf

Pace, R., Pluye, P., Bartlett, G., Macaulay, A. C., Salsberg, J., Jagosh, J., \& Seller, R. (2012). Testing the reliability and efficiency of the pilot Mixed Methods Appraisal Tool (MMAT) for systematic mixed studies review. International Journal of Nursing Studies, 49(1), 47-53. doi:10.1016/j.ijnurstu.2011.07.002

Persons, J. B. (1989). Cognitive Therapy in Practice: A Case Formulation Approach. New York, NY: W. W. Norton.

Pluye, P., Gagnon, M. P., Griffiths, F., \& Johnson-Lafleur, J. (2009). A scoring system for appraising mixed methods research, and concomitantly appraising qualitative, quantitative and mixed methods primary studies in Mixed Studies Reviews. International Journal of Nursing Studies, 46(4), 529-546. doi:10.1016/j.ijnurstu.2009.01.009

Radcliffe, K., McMullan, E., \& Ramsden, J. (2018). Developing offender manager competencies in completing case formulation: An evaluation of a training and supervision model. Probation Journal, 65(1), 27-38. doi:10.1177/0264550517744272

Ramsden, J., Lowton, M., \& Joyes, E. (2014). The impact of case formulation focussed consultation on criminal justice staff and their attitudes to work with personality disorder. Mental Health Review Journal, 19(2), 124-130. doi:10.1108/MHRJ-12-2013-0039

Shaw, J., Higgins, C., \& Quartey, C. (2017). The impact of collaborative case formulation with high risk offenders with personality disorder. The Journal of Forensic Psychiatry \& Psychology, 28(6), 777-789. doi:10.1080/14789949.2017.1324579

Shaw, J., Minoudis, P., Craissati, J., \& Bannerman, A. (2011). Developing probation staff competency for working with high risk of harm offenders with personality disorder: An evaluation of the Pathways Project. Personality and Mental Health, 6(2), 87-96. doi:10.1002/pmh.192

Skeem, J. L., Louden, J. E., Polaschek, D. L., \& Camp, J. P. (2007). Assessing relationship quality in mandated community treatment: Blending care with control. Psychological Assessment, 19, 397-410. doi:10.1037/1040-3590.19.4.397

Souto, Q. R., Khanassov, V., Pluye, P., Hong, Q. N., Bush, P., \& Vedel, I. (2014). Systematic Mixed Studies Reviews: Reliability Testing of the Mixed Methods Appraisal Tool. Paper presented at the Mixed Methods International Research Association Conference (MMIRA), Boston, USA.

Sturmey, P., \& McMurran, M. (2011). Forensic Case Formulation: Emerging Issues. In P. Sturmey \& M. McMurran (Eds.), Forensic Case Formulation (pp. 283-304). Chichester, UK: WileyBlackwell.

Sturmey, P., \& McMurran, M. (Eds.). (2011). Forensic case formulation. Chichester: Wiley-Blackwell.

Völlm, B. (2014). Case formulation in personality disordered offenders - A Delphi survey of professionals. Criminal Behaviour and Mental Health, 24(1), 60-80. doi:10.1002/cbm.1884

Whitton, C., Small, M., Lyon, H., Barker, L., \& Akiboh, M. (2016). The impact of case formulation meetings for teams. Advances in Mental Health \& Intellectual Disabilities, 10(2), 145-157. doi:10.1108/AMHID-09-2015-0044 


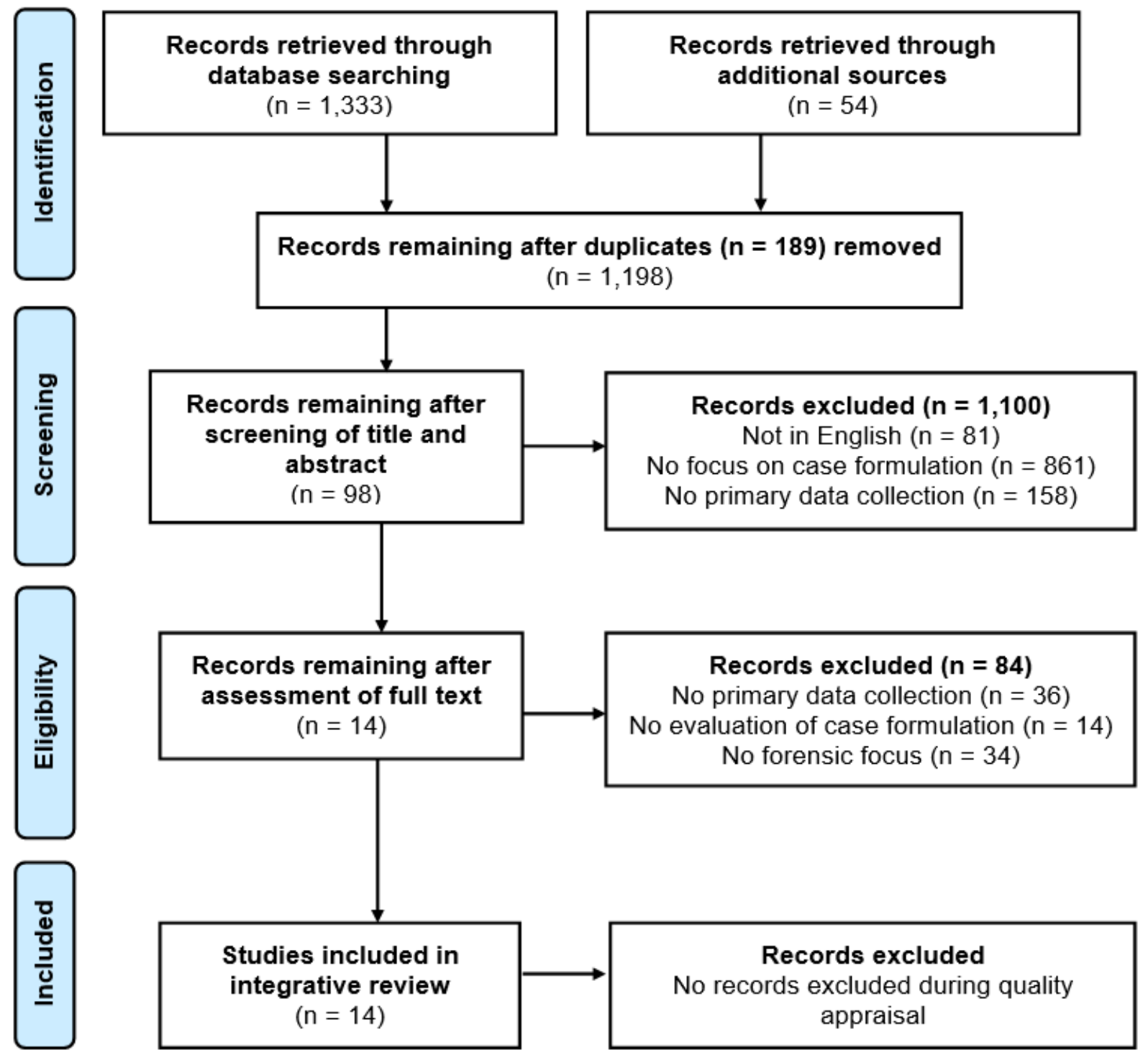

Figure 1: Study selection and screening based on PRISMA method (Moher, Liberati, Tetzlaff, Altman \& The PRISMA Group, 2009). 
Table 1.

Overview of included studies

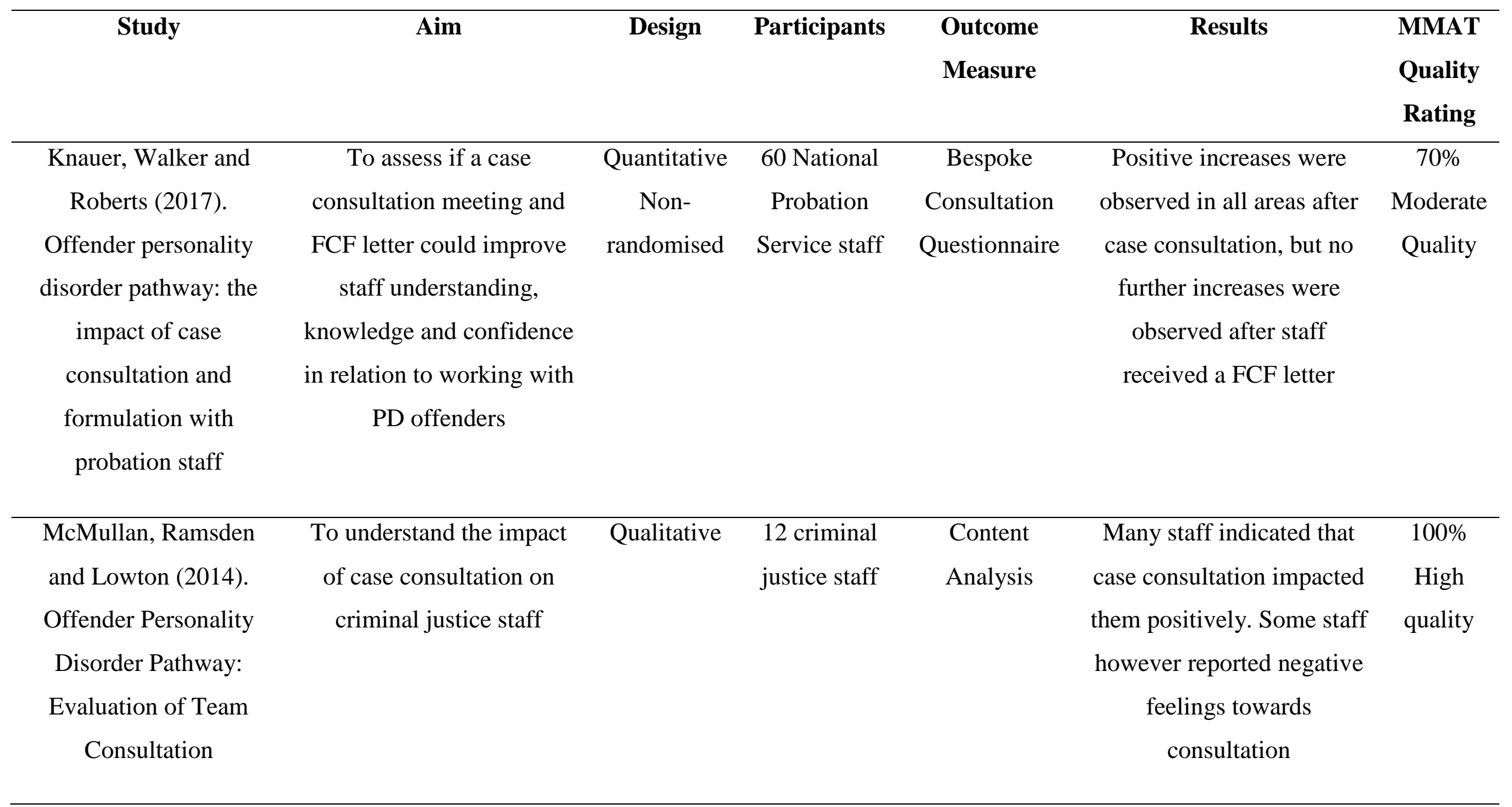


Table 1

(Continued)

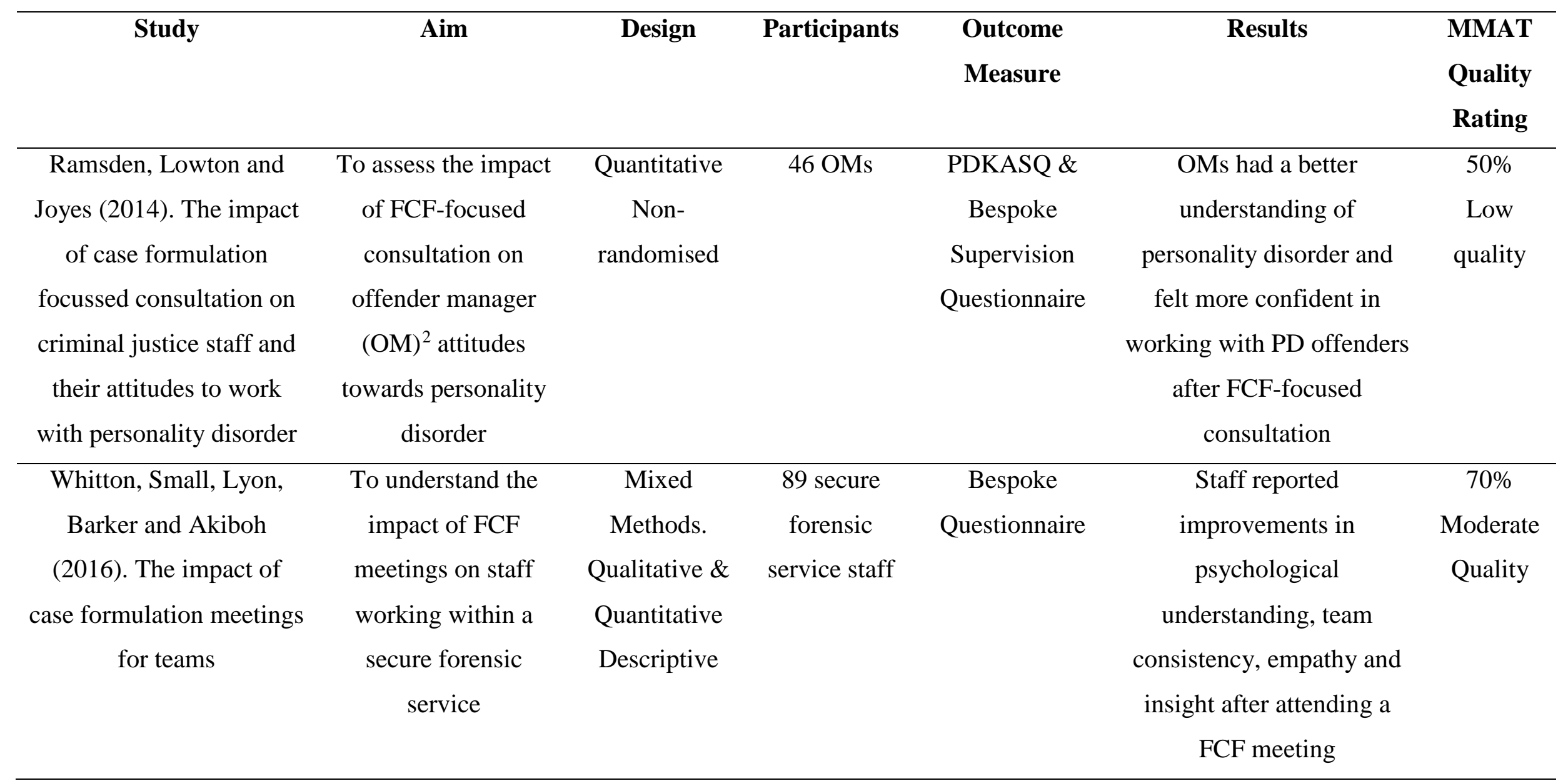


Table 1

(Continued)

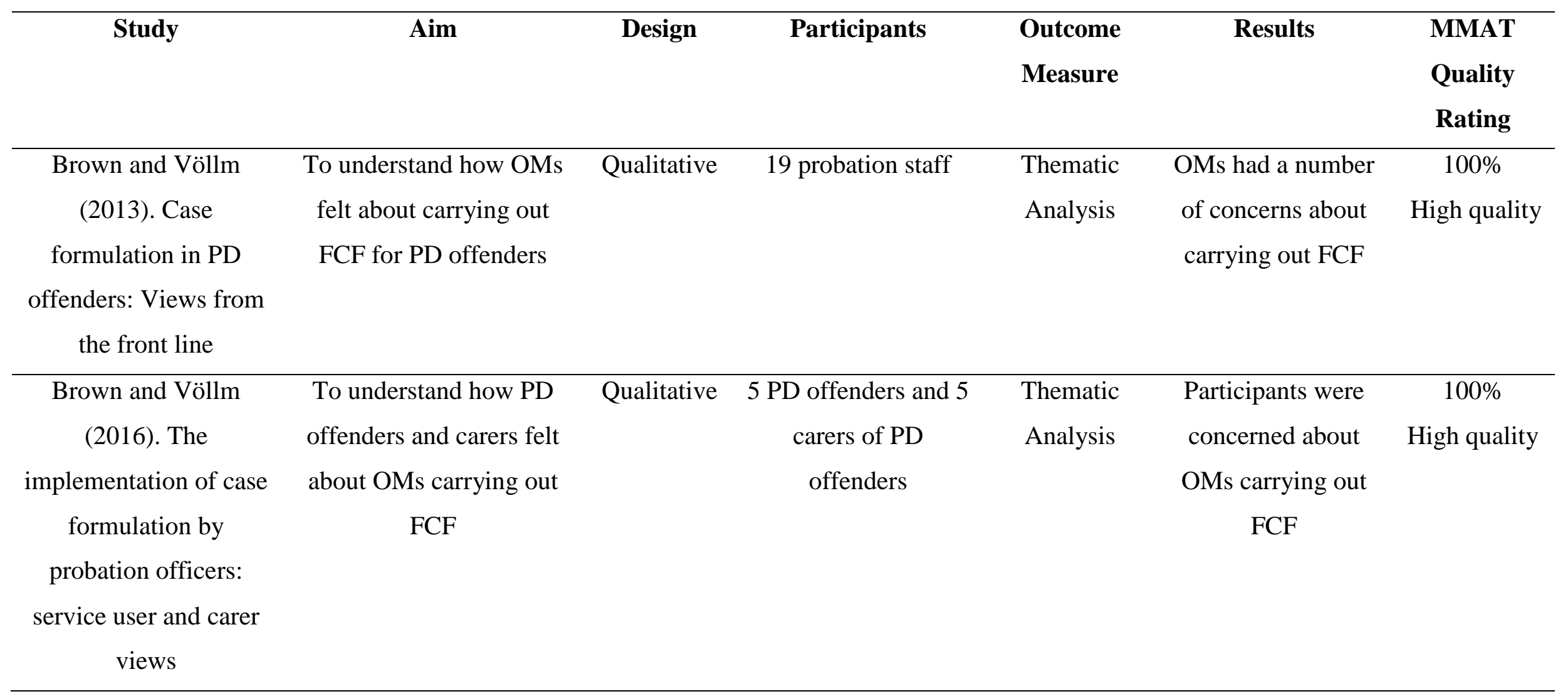


Table 1

(Continued)

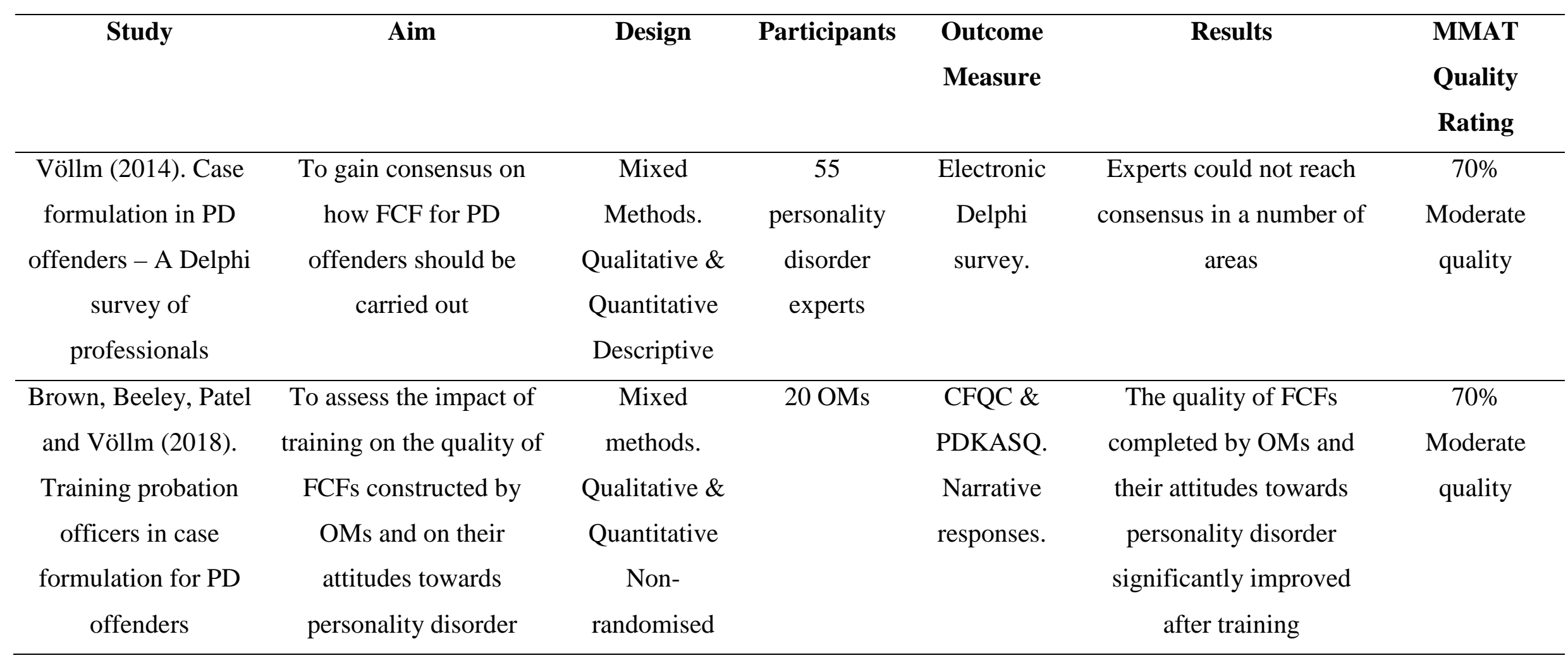


Table 1

(Continued)

\begin{tabular}{|c|c|c|c|c|c|c|}
\hline Study & Aim & Design & Participants & $\begin{array}{l}\text { Outcome } \\
\text { Measure }\end{array}$ & Results & $\begin{array}{l}\text { MMAT } \\
\text { Quality } \\
\text { Rating }\end{array}$ \\
\hline $\begin{array}{l}\text { Mapplebeck, Ramsden, } \\
\text { Lowton, Short \& Burn } \\
\text { (2017). Embedding } \\
\text { psychological thinking: } \\
\text { an evaluation of a } \\
\text { regional training model } \\
\text { for probation staff }\end{array}$ & $\begin{array}{l}\text { To assess change } \\
\text { in OM FCF skills } \\
\text { after training }\end{array}$ & Qualitative & $21 \mathrm{OMs}$ & $\begin{array}{l}\text { Thematic } \\
\text { Analysis. }\end{array}$ & $\begin{array}{l}\text { FCF skills improved } \\
\text { after training }\end{array}$ & $\begin{array}{c}90 \% \\
\text { High Quality }\end{array}$ \\
\hline $\begin{array}{l}\text { Minoudis et al., (2013). } \\
\text { An evaluation of case } \\
\text { formulation training and } \\
\text { consultation with } \\
\text { probation officers }\end{array}$ & $\begin{array}{l}\text { To assess the } \\
\text { impact of training } \\
\text { on the quality of } \\
\text { FCFs carried out } \\
\text { by OMs }\end{array}$ & $\begin{array}{l}\text { Quantitative } \\
\text { Non- } \\
\text { randomised }\end{array}$ & $64 \mathrm{OMs}$ & CFQC & $\begin{array}{l}\text { The quality of OM } \\
\text { FCFs did not } \\
\text { significantly improve } \\
\text { after training }\end{array}$ & $\begin{array}{c}60 \% \\
\text { Moderate } \\
\text { quality }\end{array}$ \\
\hline
\end{tabular}


Table 1

(Continued)

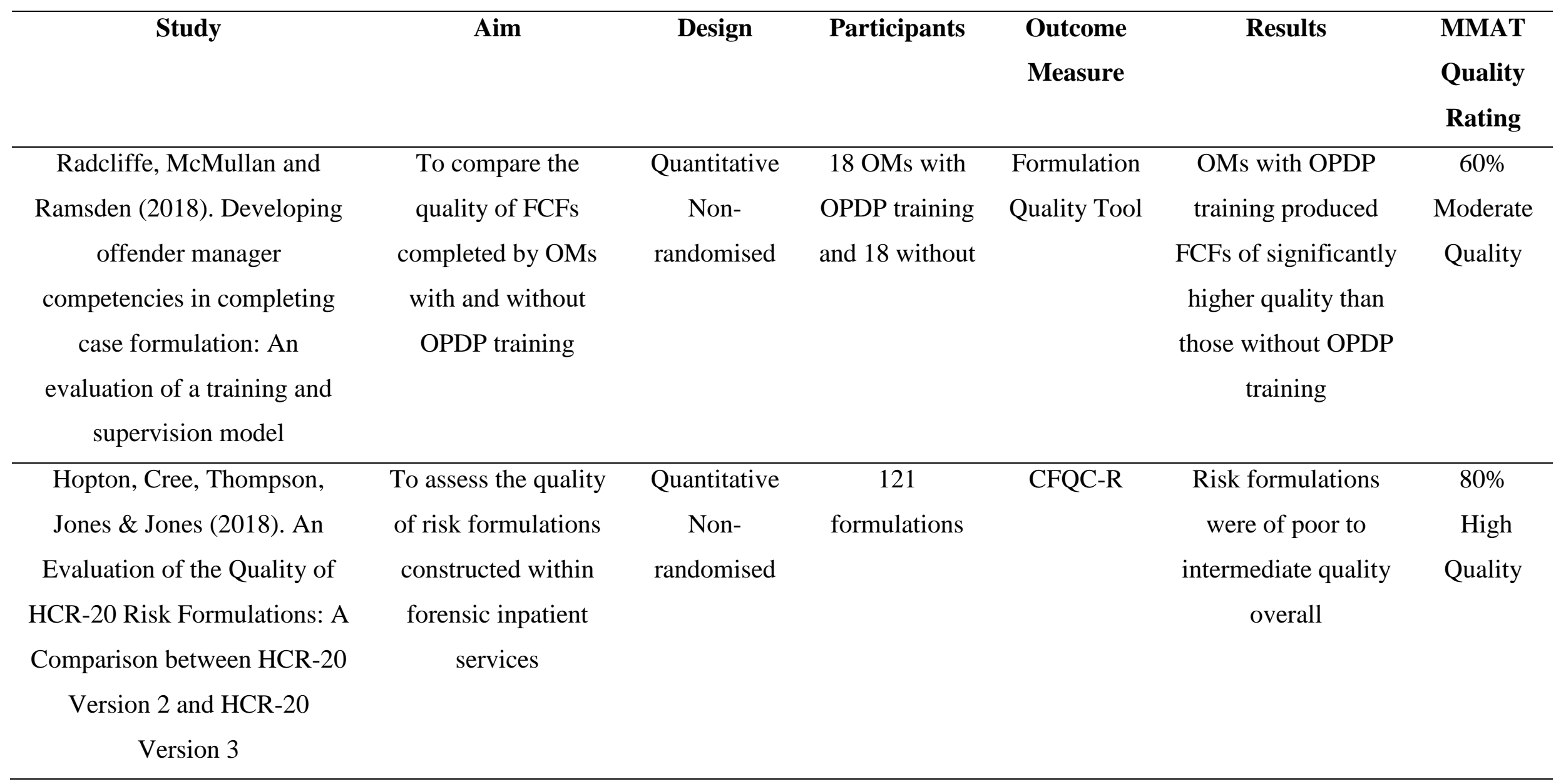


Table 1

(Continued)

\begin{tabular}{|c|c|c|c|c|c|c|}
\hline Study & Aim & Design & Participants & $\begin{array}{l}\text { Outcome } \\
\text { Measure }\end{array}$ & Results & $\begin{array}{l}\text { MMAT } \\
\text { Quality }\end{array}$ \\
\hline & & & & & & Rating \\
\hline (2016). Case formulation & from clinicians on & Methods. & psychologists & Analysis. & CFQC was useful but & High Quality \\
\hline quality checklist: a & the CFQC & Qualitative \& & / psychiatrists & & required some & \\
\hline clinicians' views & & Descriptive & & & & \\
\hline Shaw, Higgins and & To compare the & Quantitative & 39 offenders. & DRI-R and & Those in the collaborative & $60 \%$ \\
\hline Quartey (2017). The & impact of & Randomised & 77 OMs & a Perceived & condition reported & Moderate \\
\hline impact of collaborative & collaborative vs non- & Control Trial & & Benefits & significantly higher & Quality \\
\hline
\end{tabular}

Note. $\mathrm{PD}=$ Personality disordered. PDKASQ = Personality Disorder Knowledge, Attitudes and Skills Questionnaire (Bolton, Feigenbaum, Jones, Sims \& Woodward, 2010). CFQC = Case Formulation Quality Checklist (McMurran, Logan \& Hart, 2012). Formulation Quality Tool (NOMS \& NHS, 2015b). CFQC-R = Case Formulation Quality Checklist-Revised (McMurran \& Bruford, 2016). DRI-R = Dual Role Relationships Inventory - Revised (Skeem, Louden, Polaschek \& Camp, 2007). 
${ }^{2}$ The terms 'probation officer' and 'offender manager' are used interchangeably throughout the literature to describe the same role (Brown, Beeley, Patel \& Völlm, 2018). The term ‘offender manager’ (OM) will be used throughout this review to retain clarity. 
